\title{
Measurements of multiparton interactions at ATLAS
}

\author{
Lennart Adam* \\ Johannes Gutenberg University, Mainz \\ E-mail: lennart.adam@cern.ch
}

on behalf of the ATLAS Collaboration

\begin{abstract}
Measurements of multiple parton scattering in proton-proton collisions provide insight into the structure and long-range low-momentum scale interactions of the proton. In this talk we present two recent measurements using proton-proton collision data collected by the ATLAS experiment. The first measurement determines the double-parton scattering contribution to four-lepton events at $\sqrt{s}=8 \mathrm{TeV}$. An artificial neural net is used to optimise the analysis and an upper limit on the double-parton scattering fraction is set at 0.042 , which corresponds to an effective cross section of $1 \mathrm{mb}$. In the second measurement, the underlying event activity is studied in events containing a Z-boson in $\sqrt{s}=13 \mathrm{TeV}$ data. Unfolded differential cross sections are presented for charged particle multiplicity and charged particle transverse momentum in regions of azimuth measured with respect to the Z-boson direction. The data are compared to a wide variety of predictions from Monte Carlo event generators.
\end{abstract}

XXVII International Workshop on Deep-Inelastic Scattering and Related Subjects - DIS2019

8-12 April, 2019

Torino, Italy

${ }^{*}$ Speaker. 


\section{Introduction}

The parton-parton scattering at the origin of hard processes in proton-proton $(p p)$ interactions is accompanied by proton- remnant fragments that contribute to the hadronic final state through the so-called underlying event (UE). One source of the underlying-event activity, particularly in the high-energy regime of the LHC, is multi-parton interactions (MPI): interactions of pairs of partons from the interacting protons which occur simultaneously with the hard process. In high-energy $p p$ interactions, where the density of low-x partons is high, there is enough energy to produce hard multi-parton interactions. The simplest example is hard double-parton scattering (DPS), where two partons from each proton interact with each other leading to perturbative final states. This summary presents a study of the hard DPS contribution to inclusive four-lepton production in $p p$ collisions at $\sqrt{s}=8 \mathrm{TeV}$ with the ATLAS detector. The second part of the summary presents a measurement of distributions sensitive to the underlying event in inclusive $\mathrm{Z}$ boson production in $p p$ collisions at $\sqrt{s}=13 \mathrm{TeV}$.

\section{DPS in inclusive four-lepton production [1]}

The production of four-lepton (electrons or muons) final states in $p p$ interactions at $\sqrt{s}=8 \mathrm{TeV}$ is analysed for the presence of double-parton scattering, using $20.2 \mathrm{fb}^{-1}$ of data recorded by the ATLAS experiment at the LHC. Leptons with transverse momentum above 20, 15, 10 (8 if muon), and 7 (6 if muon) $\mathrm{GeV}$, sorted in descending order of $p_{T}$, are selected in the pseudorapidity range $|\eta|<2.5$ in the case of electrons and $|\eta|<2.7$ in the case of muons. The four leptons form two same-flavour opposite-charge lepton pairs. The dilepton invariant masses are required to be in the range $50<m_{\text {leading }}<120 \mathrm{GeV}$ for the leading pair and $12<m_{\text {sub-leading }}<120 \mathrm{GeV}$ for the subleading pair, where the leading pair is defined as the opposite-charge pair with invariant mass closer to the $\mathrm{Z}$ boson mass. The transverse momentum $p_{T}$ of the dileptons is required to be above $2 \mathrm{GeV}$. The events in the four-lepton invariant-mass range of $80<m_{4 l}<1000 \mathrm{GeV}$ (see also Figure 2(a)) are considered. An artificial neural network is used to discriminate between single- and doubleparton scattering events. No signal of double-parton scattering is observed and an upper limit on the fraction of the DPS contribution to the inclusive four-lepton final state of 0.042 is obtained at $95 \%$ CL. This upper limit translates, for two independent subscatterings, into a lower limit of $1.0 \mathrm{mb}$ on the effective cross section, consistent with previously measured values in different processes and at different centre-of-mass energies (Figure 2(b)).

\section{Activity of the UE in $\mathrm{Z}$ boson events [2]}

Measurements of four observables sensitive to the activity of the UE in $Z \rightarrow \mu \mu$ events are presented using $3.2 \mathrm{fb}^{-1}$ of $\sqrt{s}=13 \mathrm{TeV} p p$ collision data collected with the ATLAS detector at the LHC in 2015. Those observables are the $p_{T}$ spectrum of charged particles (see Figure 2 (a)), the number of charged particles per event ( $N_{\mathrm{ch}}$, presented in Figure 3$)$, the sum of charged-particle $p_{T}$ per event $\left(\Sigma p_{T}\right)$, and the mean of charged-particle $p_{T}$ per event $\left(\right.$ mean $\left.p_{T}\right)$. The measurement takes only stable charged particles into account with $p_{T}>0.5 \mathrm{GeV}$ and $|\eta|<2.5$

The observables are measured in different azimuthal regions of the detector relative to the $\mathrm{Z}$ boson direction, namely the transverse, trans-min , trans-max, toward and away region. This 

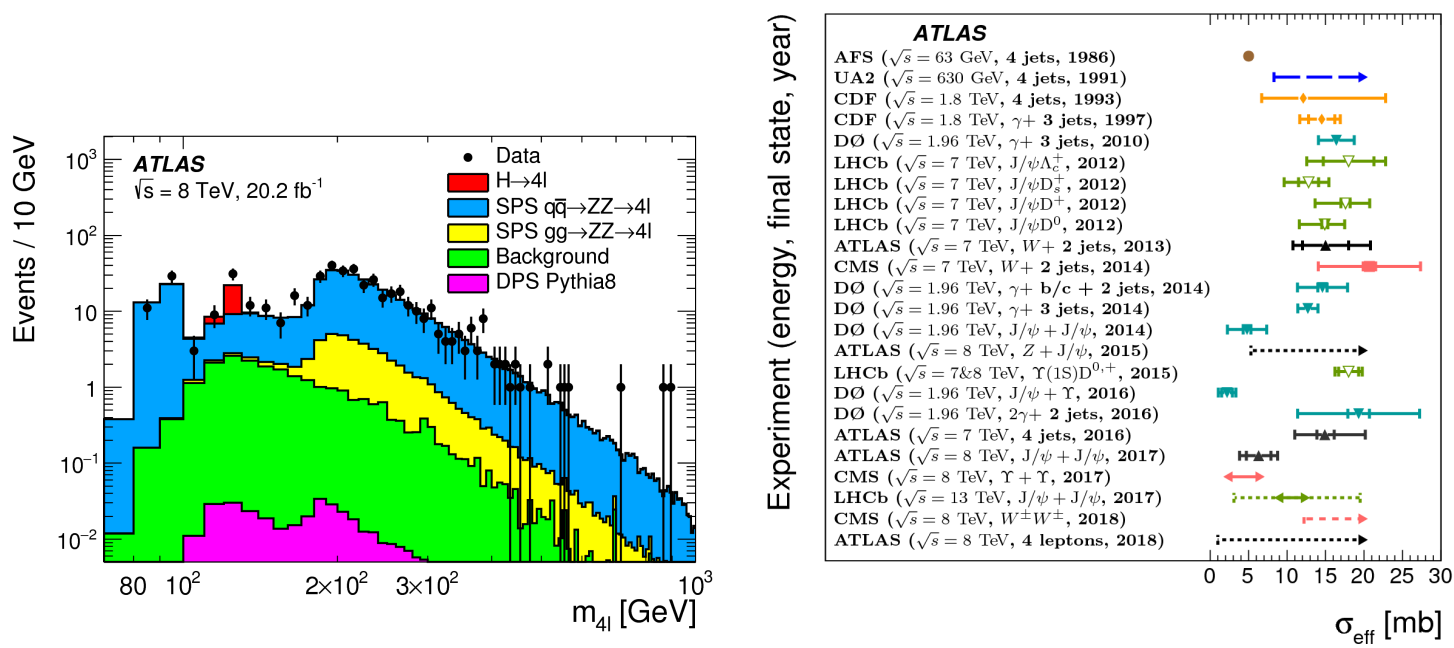

Figure 1: (a) The distribution of the four-lepton invariant mass, $m_{4 l}$. The data (black dots) are compared with the sum of signal and background MC expectations (filled coloured histograms). Also shown is the expected contribution of DPS from Pythia 8. (b) Summary of measurements and limits on the effective cross section, determined in different experiments sorted chronologically. The measurements that were made by different experiments are denoted by different symbols and colours. The inner error bars represent statistical uncertainties and the outer error bars correspond to the total uncertainty. Dashed arrows indicate lower limits. Lines with arrows on both ends represent ranges of the effective cross-section values, determined within a single publication. In the case of the double $J / \Psi$ measurement by $\mathrm{LHCb}$, the dashed line denotes the upper and lower uncertainties. The AFS measurement, indicated with a dot, was published without uncertainties.

summary focuses on the trans-min region, because the contribution from the hard scattering is suppressed by construction. The distributions are further distinguished in intervals of the $\mathrm{Z}$ boson $p_{T}$ and for two regions of transverse thrust $T_{\perp}$ [3]. Transverse thrust characterizes the topology of the tracks in the event. Transverse thrust has a maximum value of 1 for a pencil-like dijet topology and a minimum value of $2 / \pi$ for a circularly symmetric distribution of particles in the transverse plane. As proposed in Ref. [4], events with lower values of $T_{\perp}$ are more sensitive to the MPI component of the UE. The two regions of thrust examined are $T_{\perp} \leq 0.75$ and $T_{\perp}>0.75$, which are optimized to distinguish extra jet activity from the actual UE activity.

All results are presented on particle level. The Powheg+Pythia8 sample is used to unfold via Bayesian Iterative Unfolding to the bare level quantites of the muons. The following sources of uncertainties are considered (in falling order of their contribution to the total uncertainty): inaccuracies of the detector and pile-up modelling, the dependence of the unfolding on the prior knowledge, background contributions as well as statistical uncertainties.

The predictions from three Monte Carlo generators (POWheg+Pythia8, Sherpa and HerWIG++) are compared with the data. In general, the qualitative behaviour of the UE activity matches the predictions, but all tested generators and tunes show significant deviations from the data distributions regardless of the observable.

The most striking difference between the different regions in $T_{\perp}$ is observed for the PowHEG+PYTHIA8 generator when focusing on the low $p_{T}^{Z}$ bins for $N_{\mathrm{ch}}$ as presented in Figure 3. In MPI-sensitive 
regions (left plot in Figure 3 ) the distribution of $N_{\mathrm{ch}}$ by POWHEG+PYTHIA 8 is shifted towards higher numbers of charged-particles relative to the data while for the region of high $T_{\perp}$ (right plot of Figure 3) the the generator tends to lower values of $N_{\mathrm{ch}}$. The regions of high values of $T_{\perp}$ are dominated by extra jet activity which is not adequately modelled in POWHEG+PYTHIA8.

Finally, a selection of the results are compared to previous measurements at lower centre-ofmass engeries. With higher centre-of-mass energies, more energy is available for the processes forming the UE e.g. MPI. Hence, the rise of the UE activity as a function of $\sqrt{s}$ is expected (see Figure 2).
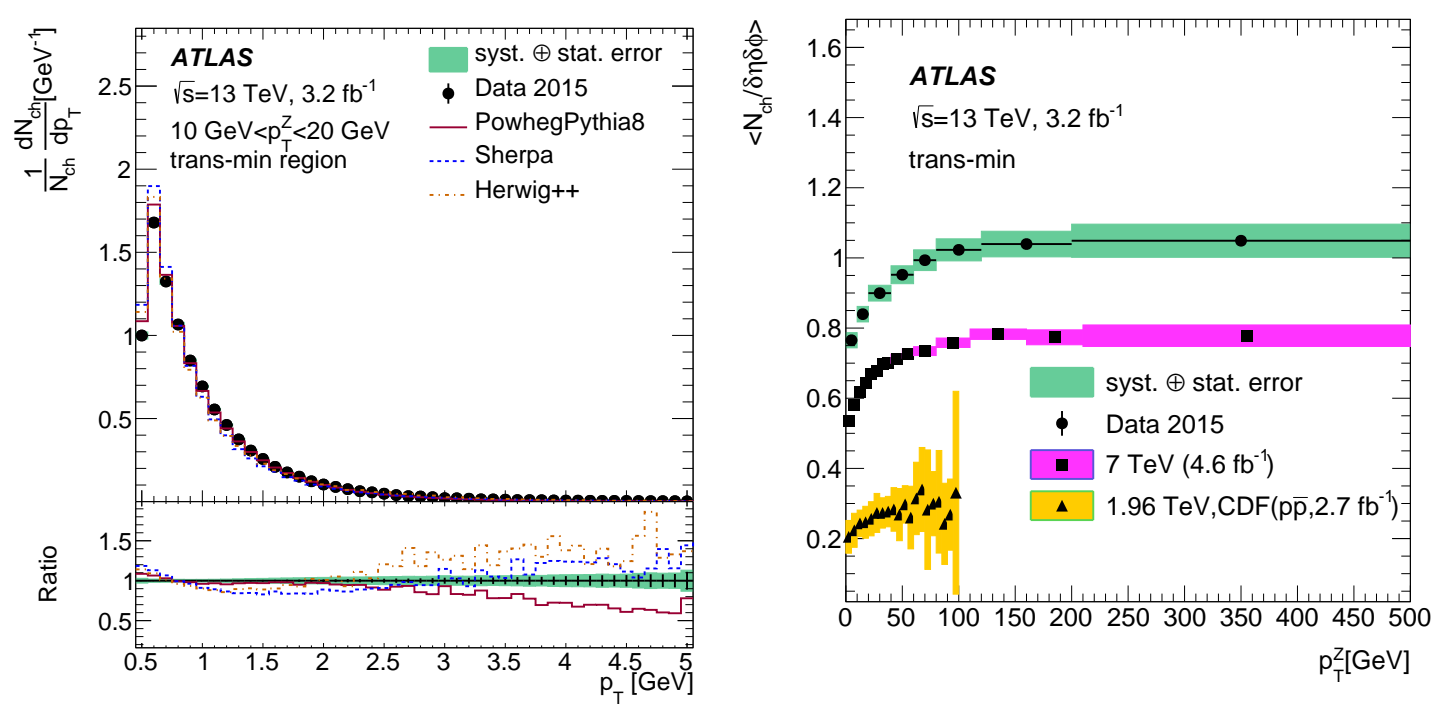

Figure 2: (a) Measured spectra of $p_{T}$ in the trans-min region inclusively in $T_{\perp}$ for events with $10<p_{T}^{Z}<$ $20 \mathrm{GeV}$. Predictions of Powheg+Pythia8, Sherpa. and Herwig++ are compared with the data. The ratios shown are predictions over data. (b) The distributions of $\left\langle N_{\mathrm{ch}}\right\rangle$ measured at $\sqrt{s}=13 \mathrm{TeV}$ compared with the results of the previous ATLAS measurements at $\sqrt{s}=7 \mathrm{TeV}$ [5] and the CDF measurements at $\sqrt{s}=1.96 \mathrm{TeV}$ [6]. The error bars correspond to the full uncertainties of the corresponding measurement. 

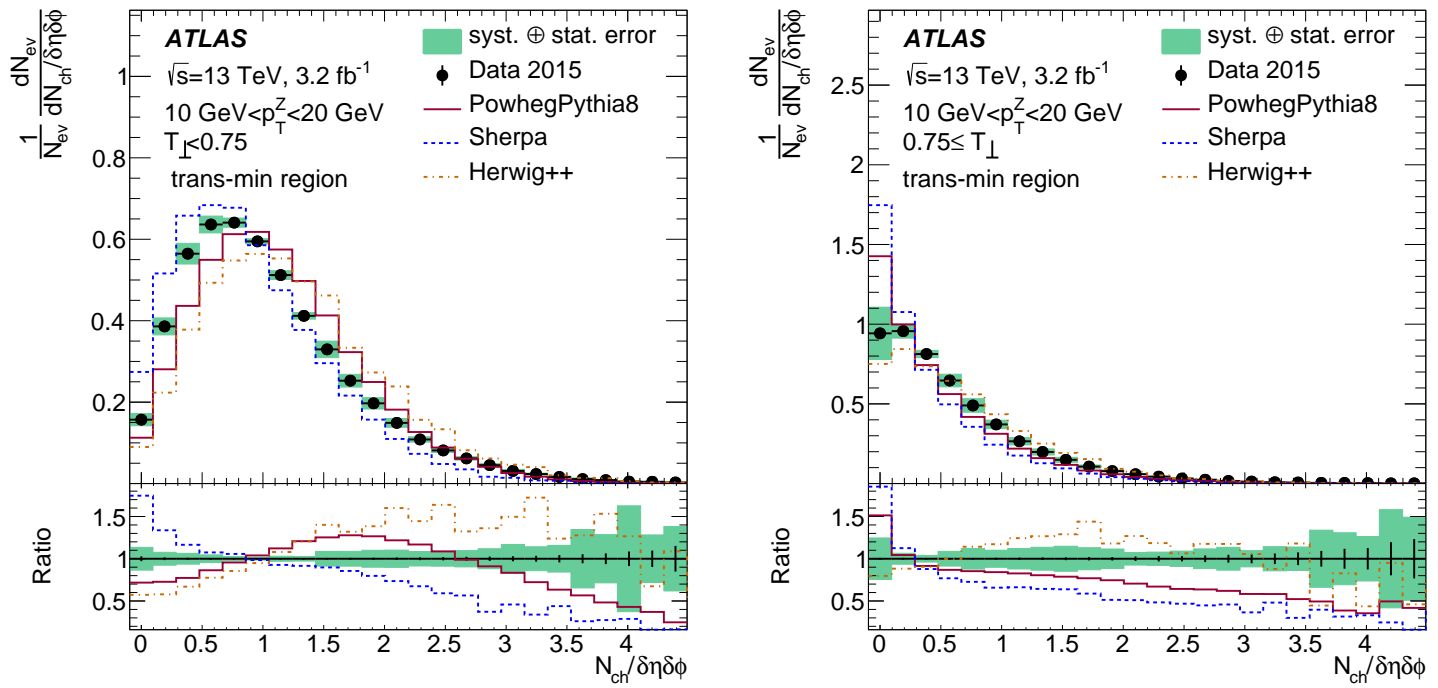

Figure 3: Measured number of charged particles in the trans-min region for $T_{\perp}<0.75$ (left) and $0.75 \leq T_{\perp}$ (right) for events with $10<p_{T}^{Z}<20 \mathrm{GeV}$. Predictions of PowheG+Pythia8, Sherpa, and HerwiG++ are compared with the data. The ratios shown are predictions over data. 


\section{References}

[1] ATLAS Collaboration, Study of the hard double-parton scattering contribution to inclusive four-lepton production in pp collisions at $\sqrt{s}=8 \mathrm{TeV}$ with the ATLAS detector, Phys. Lett. B 790 (2019) 595 [1811.11094].

[2] ATLAS Collaboration, Measurement of distributions sensitive to the underlying event in inclusive $Z$ boson production pp collisions at $\sqrt{s}=13 \mathrm{TeV}$ with the ATLAS detector, 1905.09752 .

[3] A. Banfi, G. P. Salam and G. Zanderighi, Phenomenology of event shapes at hadron colliders, JHEP 06 (2010) 038 [1001.4082].

[4] D. Kar and D. S. Rafanoharana, Probing underlying event in Z-boson events using event shape observables, Int. J. Mod. Phys. A 34 (2018) 1950022 [1801.05218].

[5] ATLAS Collaboration, Measurement of distributions sensitive to the underlying event in inclusive Z-boson production in pp collisions at $\sqrt{s}=7 \mathrm{TeV}$ with the ATLAS detector, Eur. Phys. J. C 74 (2014) 3195 [1409.3433].

[6] CDF collaboration, Studying the underlying event in Drell-Yan and high transverse momentum jet production at the Tevatron, Phys. Rev. D 82 (2010) 034001 [1003.3146]. 